\title{
Mitteilungen der DGIM
}

Diabetologe 2018 $\cdot 14: 278$

https://doi.org/10.1007/s11428-018-0358-3

๑) Springer Medizin Verlag GmbH, ein Teil von Springer Nature 2018

Deutsche Gesellschaft für Innere Medizin e.V.

\section{Redaktion}

Prof. Dr. Dr. h.c. Ulrich R. Fölsch (v.i.S.d.P.)

\section{Korrespondenzadresse}

Deutsche Gesellschaft für Innere Medizin e.V. (DGIM)

Irenenstraße 1

D-65189 Wiesbaden

Tel. 0611/205 8040-0 Fax 0611/205 8040-46

info@dgim.de

\section{European Congress of Internal Medicine (ECIM), 30. August bis 1 . September 2018 im RheinMain CongressCenter (RMCC), Wiesbaden}

\section{Internal Medicine goes Europe}

In der Kongress-Landschaft der Inneren Medizin bildet der 17 . ECIM Kongress vom 30. August bis 1. September 2018 im neu eröffneten RheinMain CongressCenter (RMCC) in Wiesbaden, die Plattform zum Austausch der Internisten aus ganz Europa. Der unter dem Dach der European Federation of Internal Medicine (EFIM) stattfindende Kongress repräsentiert 35 nationale internistische Fachgesellschaften aus 33 Mitgliedsländern von Algerien bis United Kingdom: Eine ideale Gelegenheit für Praktiker, Kliniker, Forscher und junge Internisten, sich dort auf einen aktuellen Stand zu bringen, sich auszutauschen und zu vernetzen.

\section{Das Hauptprogramm ist online}

Unter dem Leitthema ,The art of managing clinical complexity: an integrated patient-centred approach" bietet der von Kongress-Präsidentin Prof. Dr. med. Petra-Maria Schumm-Draeger aus München gestaltete ECIMKongress 2018 ein breites Fortbildungsprogramm mit Inhalten, die alle europäischen Internisten derzeit bewegen: von den Chancen und Risiken digitaler Anwendungen in der Medizin über Multimorbidität und Geriatrie, Stoffwechselerkrankungen bis hin zum Umgang mit dem überall wachsenden ökonomischen Druck bei der Behandlung von Patienten.

Namhafte internationale Experten sprechen in vier Plenar-

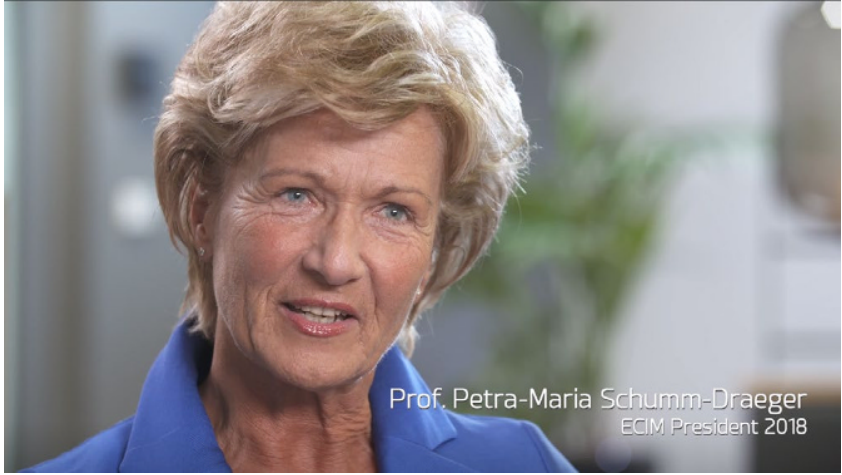

$\Delta$ Im Kongress-Video gibt Kongress-Präsidentin Professor Petra-Maria Schumm-Draeger aus München einen Ausblick auf die Highlights des ECIM 2018. Das Video ist abrufbar unter www.ecim2018.eu

sitzungen über Digitalisierung, High Value Care, Möglichkeiten, den Adipositas-Tsunami zu stoppen und Störungen des CalciumStoffwechsels.

Vor dem eigentlichen Hauptprogramm finden bereits am Donnerstag, 30. August 2018 verschiedene Pre-Congress Ultraschall-Workshops statt. Ebenso vorab starten der halbtägige Teaching Course der Obesity Management Task Force (OMTF) sowie die Sitzung „Less is more".

\section{Frühbucherrabatt bis zum 4. Juli 2018 und Sonder- konditionen nutzen}

Neben den regulären Tickets sind Tageskarten erhältlich. Sonderkonditionen gelten für Studenten und Junge Internisten unter 35 Jahren. Der Frühbucherrabatt gilt bis zum 4. Juli 2018.

Mehr Informationen zum ECIM 2018 finden Sie unter www.ecim2018.eu. 